\title{
MANIFESTAÇÕES CUTÂNEAS RELACIONADAS À COVID-19: UMA REVISÃO DA LITERATURA
}

\section{CUTANEOUS MANIFESTATIONS RELATED TO COVID-19: A LITERATURE REVIEW}

\author{
Ana Paula Vedana Marin ${ }^{1}$, Arieli Acatrolli Fucks ${ }^{1}$, \\ Gabriel Tarasconi Zanin ${ }^{1}$, Laura Piovezani ${ }^{1}$, Vanessa Guse ${ }^{1}$, \\ Yasmin Dalla Vecchia de Souza ${ }^{1}$, Gilvana Aparecida Bonella ${ }^{1}$
}

\section{RESUMO}

Neste artigo identificamos as manifestações de pele mais comuns relacionadas à doença do coronavírus 19 (COVID-19), a fim de facilitar o diagnóstico realizado pelos profissionais da saúde. Foram analisados um total de 524 pacientes, por meio de 20 artigos, sobre a relação entre o vírus e as lesões de pele. Os artigos considerados elegíveis para essa revisão preencheram critérios como descrição completa das lesões cutâneas e presença de foto. A prevalência das lesões de pele aumentou com a idade, sendo maior no sexo feminino. Observou-se cinco principais tipos de manifestações, em ordem decrescente de prevalência: exantema maculopapular generalizado morbiforme, erupção cutânea papulovesicular, urticária, livedo reticular e placas eritematosas. Esses padrões aparecem em momentos diferentes da doença e estão associados a diferentes durações, gravidade e prognóstico. Vale destacar, que grande parte das lesões foram encontradas em tronco e extremidades. Além dessas manifestações, outros sintomas foram identificados, sendo febre o sintoma mais comum. Dessa forma, compreender as diversas apresentações do coronavírus da síndrome respiratória aguda grave 2 (SARS-CoV-2) é de extrema importância no entendimento epidemiológico da doença. Portanto, profissionais da saúde devem atentar para as manifestações cutâneas nos pacientes pois, em conjunto com outros sinais e sintomas, ajudam a compor o quadro clínico da COVID-19, colaborando para um diagnóstico clínico e diferencial precoce.

Palavras-chave: COVID-19; dermatologia; exantema; lesão; pele; urticária

\begin{abstract}
In this article, we identified the most common skin manifestations related to coronavirus disease 2019 (COVID-19) to facilitate the diagnosis made by health professionals. A total of 524 patients were included by analyzing 20 articles regarding the relation between the virus and skin lesions. The articles considered eligible for this review met criteria such as complete description of the skin lesions and presence of a photograph. The prevalence of skin lesions increased with age, being higher in women. The following five main types of manifestations were observed, in descending order of prevalence: generalized morbilliform maculopapular rash, papulovesicular rash, urticaria, livedo reticularis and erythematous plaques. These patterns appeared at different times of the disease and were associated with different durations, severity and prognosis. It is worth noting that many lesions were found on the trunk and extremities. In addition to these manifestations, other symptoms were identified, with fever being the most common. Thus, understanding the various presentations of severe acute respiratory syndrome coronavirus 2 (SARS-CoV-2) is extremely important in the epidemiological understanding of the disease. Therefore, health professionals should pay attention to skin manifestations in patients because, together with other signs and symptoms, they help formulate the clinical picture of COVID-19, contributing to an early clinical and differential diagnosis.
\end{abstract}

Keywords: COVID-19; dermatology; rash; lesion; skin; urticaria
Clin Biomed Res. 2020;40(4):223-234

1 Faculdade de Medicina, Universidade de Passo Fundo. Passo Fundo, RS, Brasil.

Autor correspondente:

Gilvana Aparecida Bonella

bonella@upf.br

Faculdade de Medicina, Universidade de Passo Fundo

Av. Brasil Leste, 285

99052-900, Passo Fundo, RS, Brasil. 


\section{INTRODUÇÃO}

A doença do coronavírus 19 (COVID-19), declarada pandêmica, acomete principalmente o sistema respiratório, produzido febre e sintomas gripais. No entanto, uma série de relatos tem documentado outras manifestações relacionadas a infecção pelo SARS-CoV-2, inclusive manifestações cutâneas. Diversas lesões de pele relacionadas à COVID-19 já foram documentadas; contudo, apesar de relatos de casos se acumularem na literatura, o conhecimento sobre o envolvimento do sistema tegumentar ainda é limitado e pouco difundido. Diante disso e do fato de que a COVID-19 requer um diagnóstico rápido $e$ preciso, essa revisão de literatura visa estabelecer um padrão do acometimento cutâneo e listar as manifestações de pele mais comuns, a fim de facilitar o diagnóstico diferencial de pacientes que apresentem queixas dermatológicas após terem apresentado sintomas gripais.

\section{MÉTODOS}

A pesquisa de literatura foi realizada através de duas plataformas em busca de artigos originais e artigos de revisão publicados desde o início da pandemia até maio de 2020. Na plataforma PUBMED os termos utilizados para a busca foram: "COVID-19" e "SARSCov-2" em combinação com os termos "dermatology", "cutaneous", "urticaria", "rash" e "skin". Já na plataforma PubCovid-19, a qual indexa diariamente artigos publicados sobre COVID-19 classificando-os nas principais áreas médicas, buscamos artigos presentes na categoria "dermatologia".

Os artigos considerados elegíveis para essa revisão preencheram os seguintes critérios de inclusão: presença de descrição completa das lesões cutâneas relatadas e presença de foto das mesmas. Além disso, priorizamos aqueles que tiveram relato da realização de biopsia das lesões de pele suspeitas. Os artigos que não demonstrassem relatos de lesões de pele em pacientes com COVID-19 ou não apresentassem conteúdo relevante para o presente artigo foram desconsiderados. Os dados coletados nos artigos selecionados foram organizados na Tabela 1 contendo: autor, ano de publicação, local, número de pacientes, idade, sexo, suspeita ou confirmação para COVID-19, descrição das lesões cutâneas, local das lesões, tempo de aparecimento, sintomas associados, descrição da biopsia e tratamento realizado. 


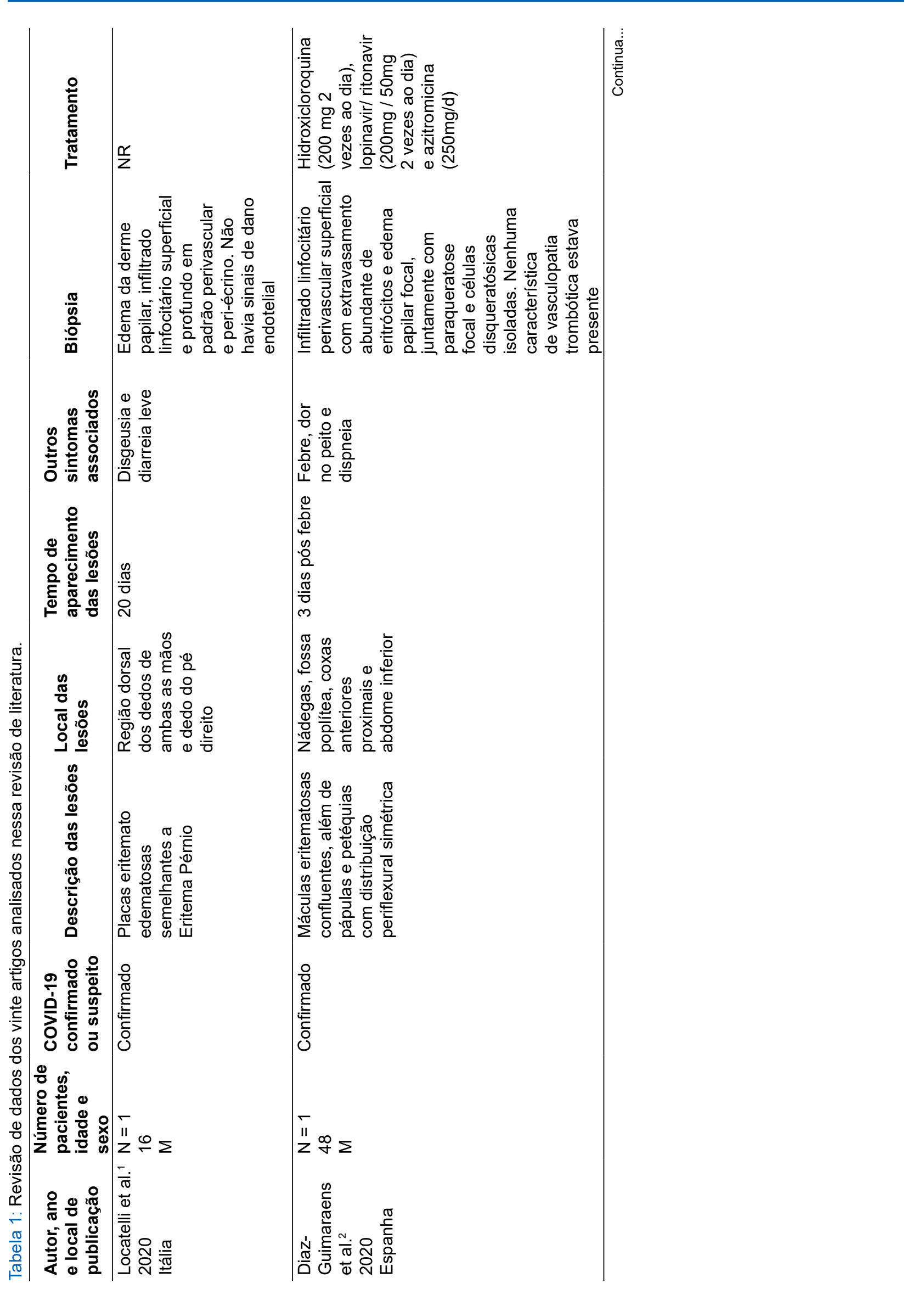




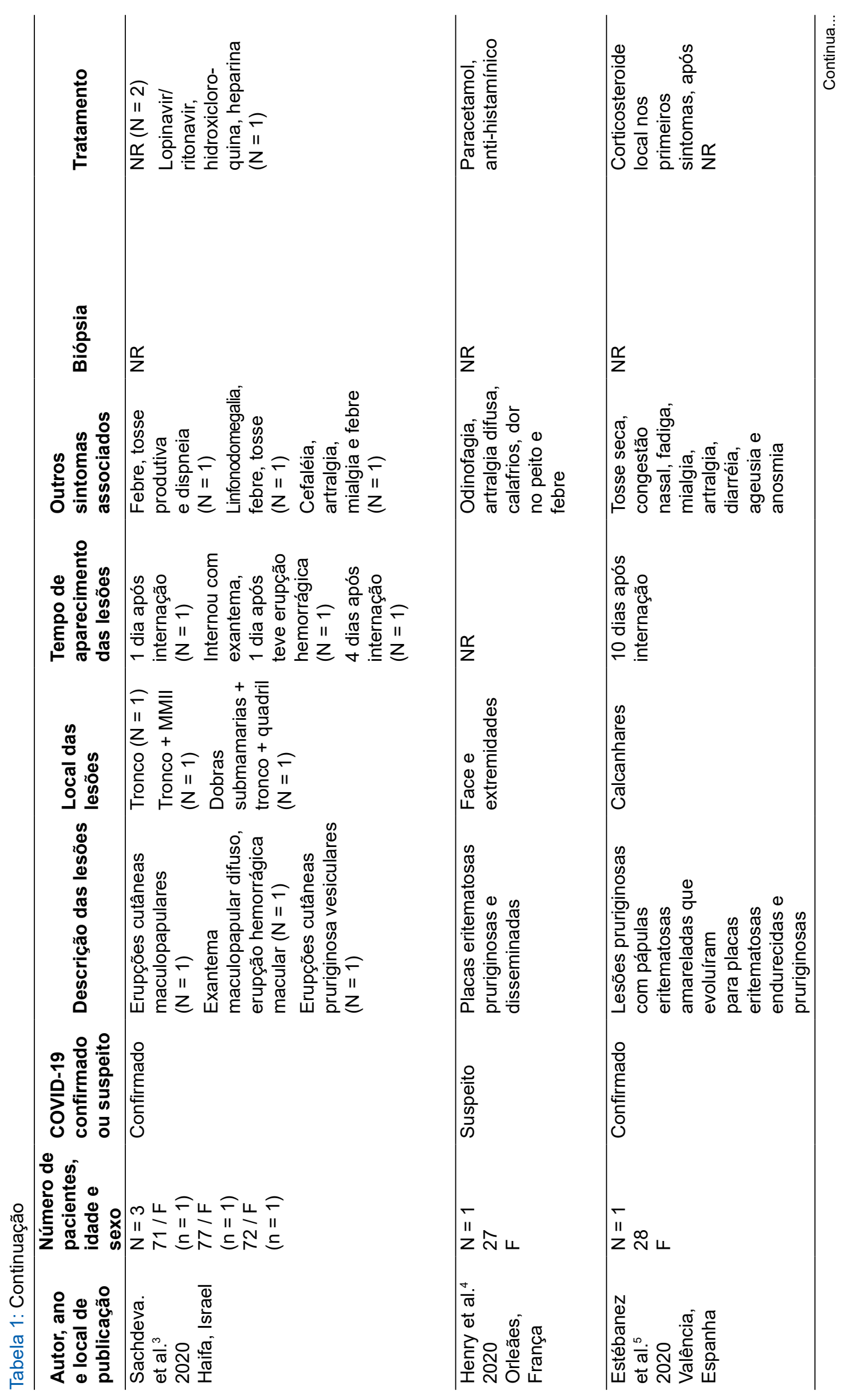




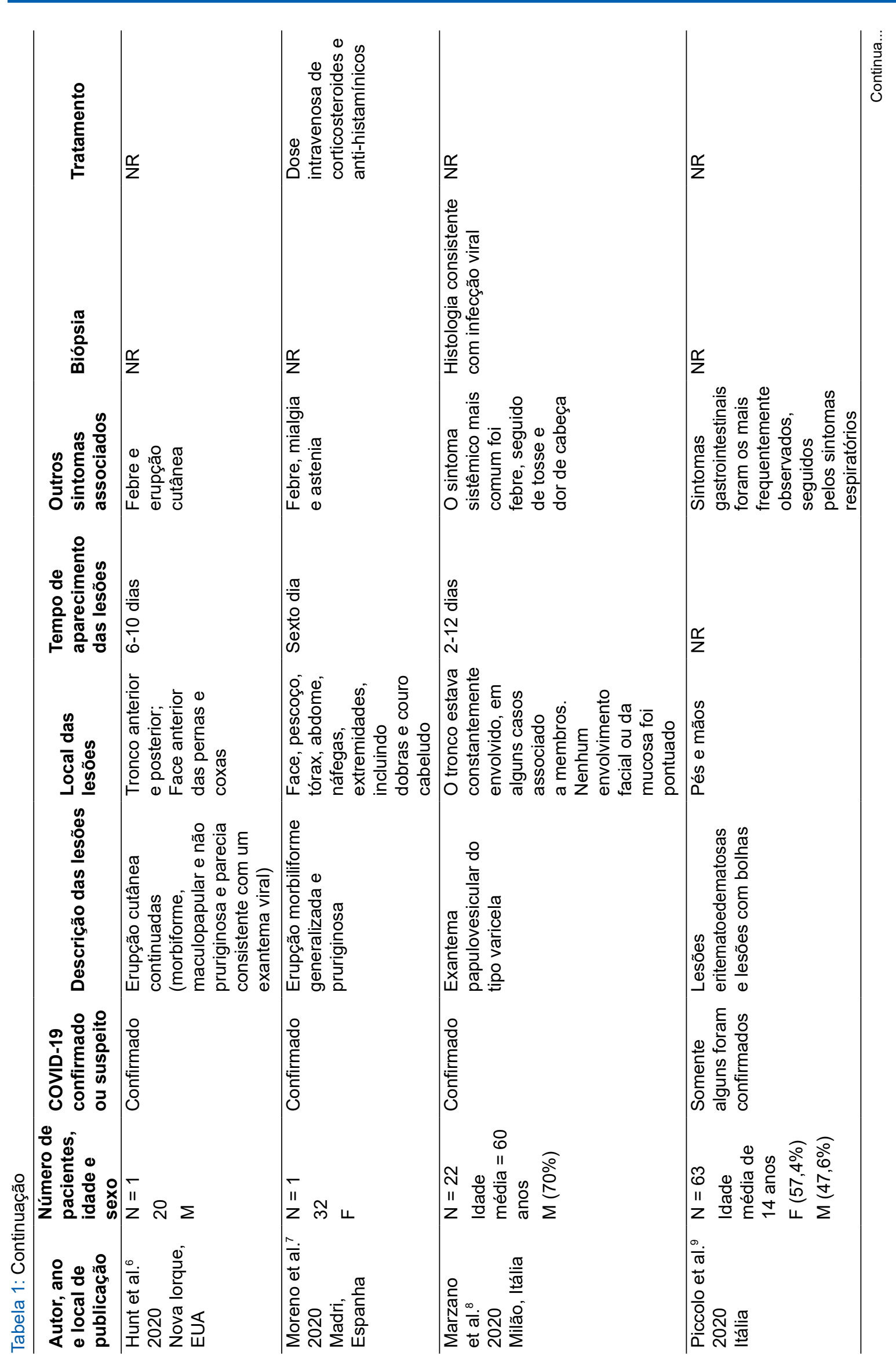




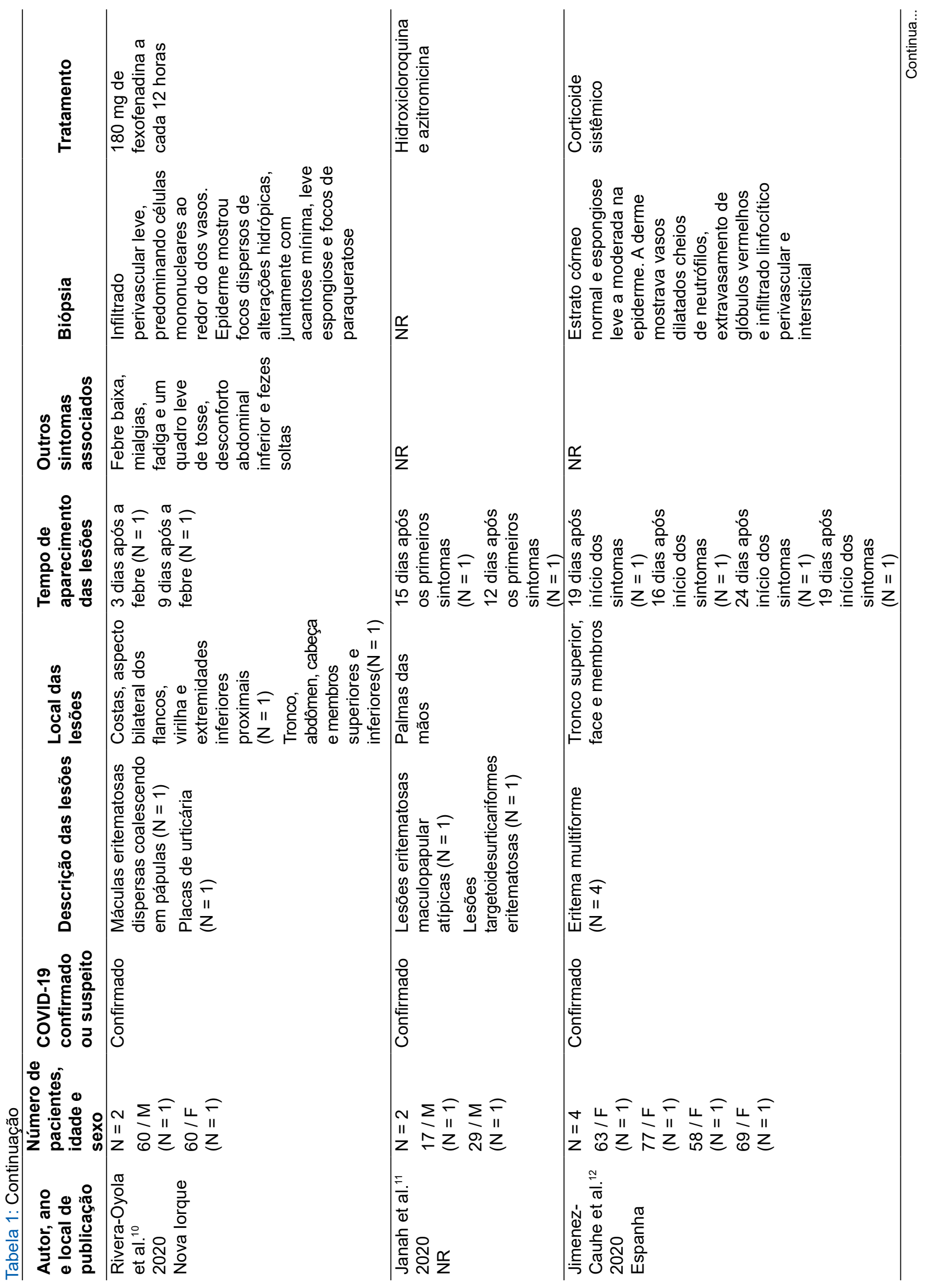




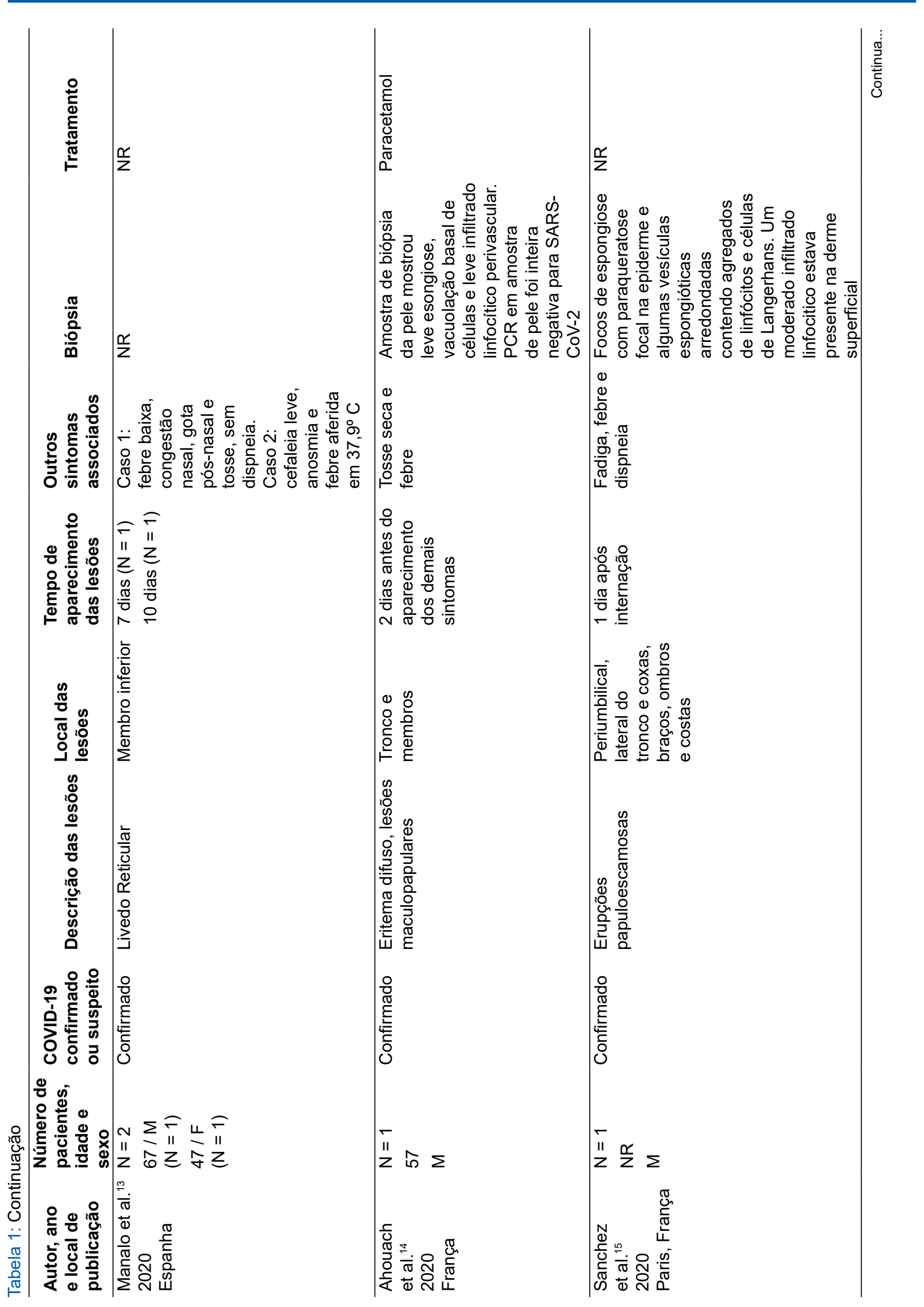




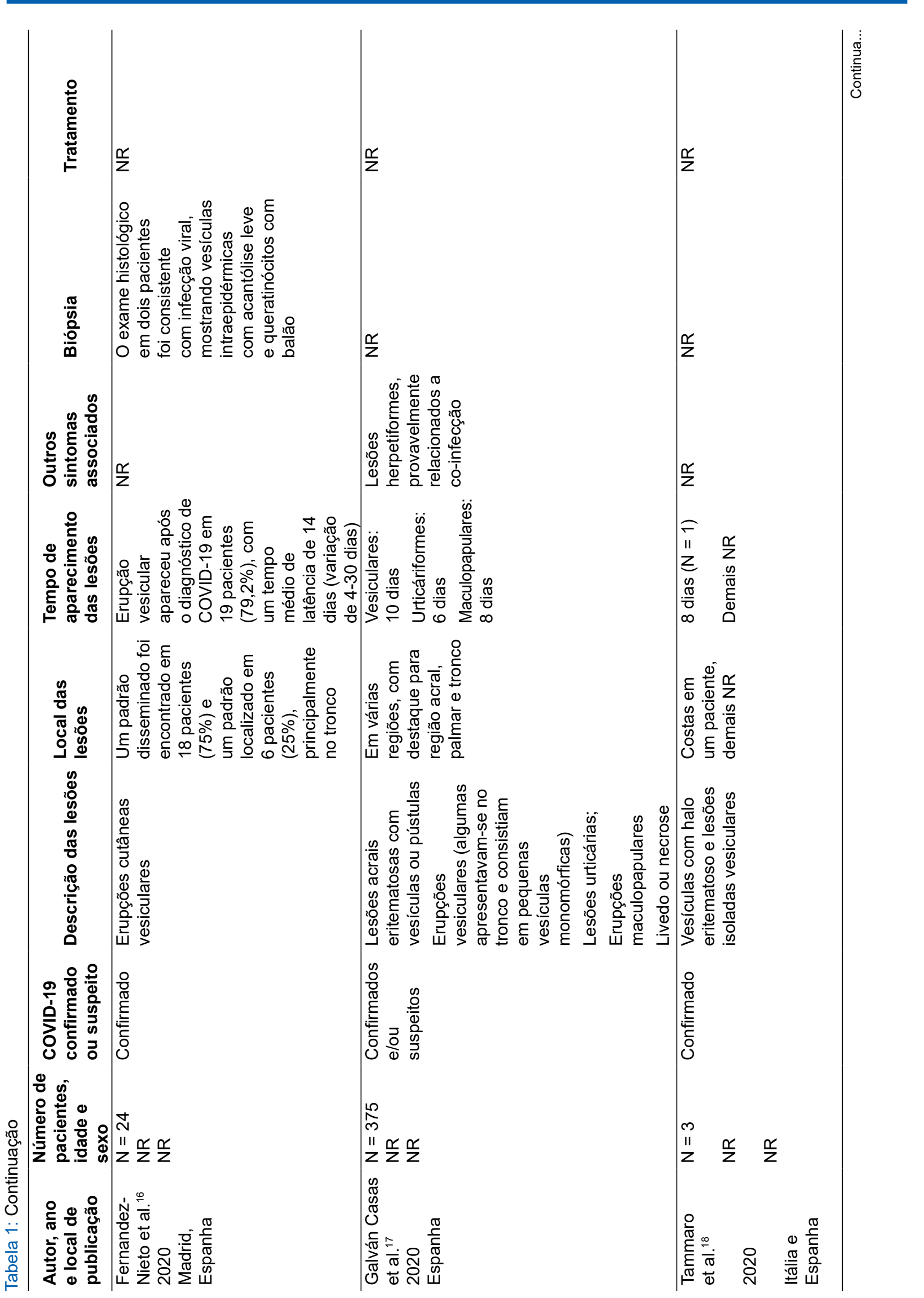




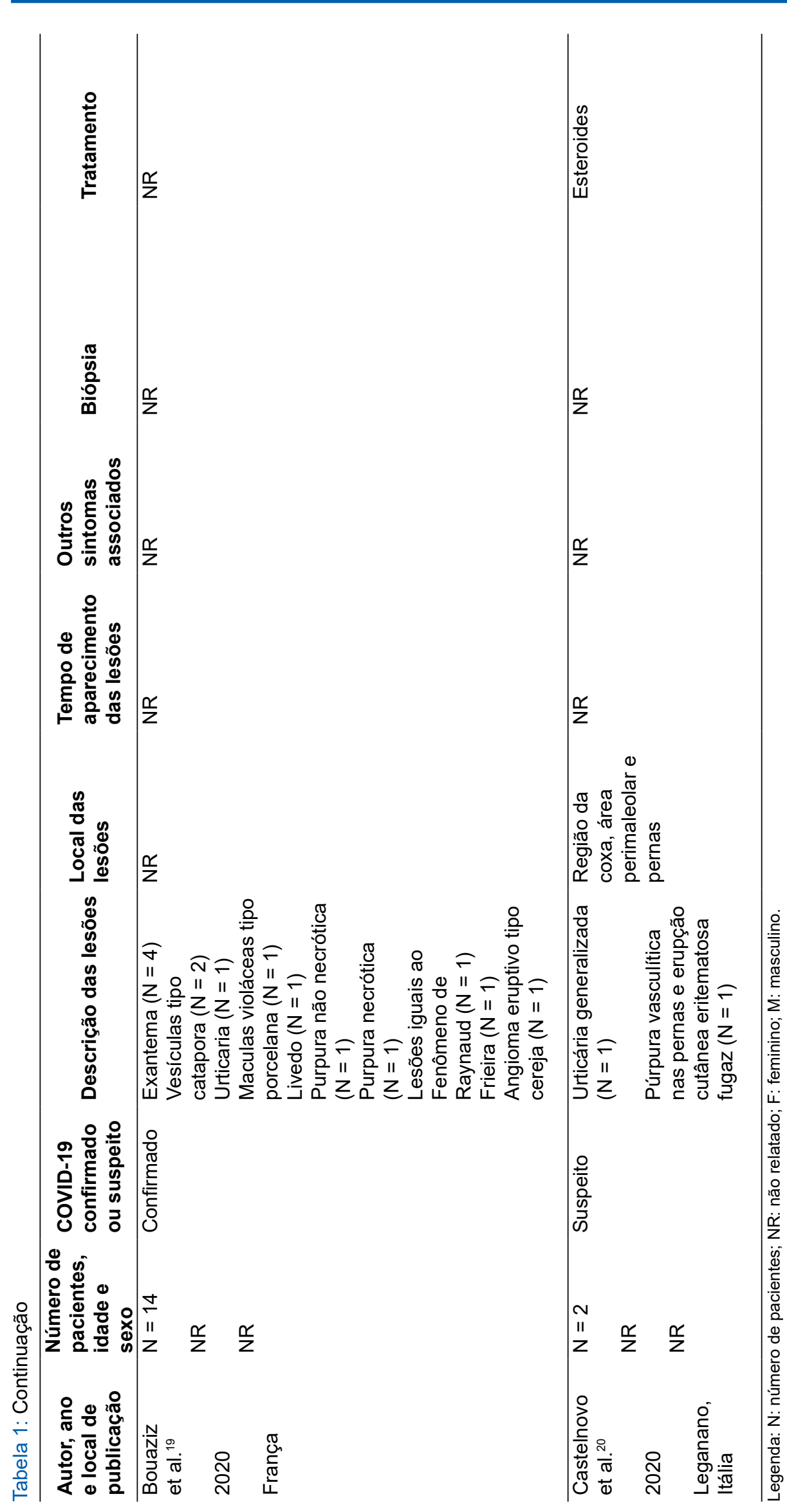




\section{RESULTADOS}

Nesse estudo foi analisado um total de 524 pacientes, através de 20 artigos sobre a relação entre COVID-19 e lesões de pele. A média de idade foi 30,3 anos (faixa de 14 a 77 anos). Sendo que apenas $20 \%$ (105/524) desses pacientes tiveram a idade descrita ${ }^{1-14}$. A prevalência do desfecho aumentou com a idade, sendo maior no sexo feminino (51,8\% mulheres e $48,2 \%$ homens). Somente 20,2\% (106/524) dos casos foi descrito o sexo $0^{1-15}$

O tempo médio do início de aparecimento das lesões cutâneas foi de 8,5 dias (variação de 2 a 24 dias). Dado relatado em 84,7\% (444/524) dos casos $^{1-3,5-8,10-18}$.

Em termos de diagnóstico COVID-19, todos os casos tiveram essa informação validada, havia suspeita em 4,96\% (26/524) dos pacientes ${ }^{4,17,20}$ e $95,04 \%$ (498/524) foram confirmados ${ }^{1-3,5-19}$.

Todos os pacientes tiveram as lesões cutâneas descritas, observando-se vários padrões. A manifestação cutânea mais comum causada pelo COVID-19 foi o exantema maculopapular generalizado morbiforme, com 40\% (210/524) dos pacientes apresentando essa lesão de pele $2,3,6,7,10,11,14,15,17,19$. Foi observada erupção cutânea papulovesicular em 30\% (157/524) dos pacientes ${ }^{3,8,16-19}$. Ocorreu urticária em 14,3\% (75/524) dos pacientes relatados ${ }^{4,10,17,19,20}$, e presença de livedo reticular foi observada em 4,6\% (24/524) dos pacientes em geral ${ }^{13,17,19}$. Por fim, 7\% (37/524) dos pacientes apresentaram placas eritematoedematosas semelhantes a eritema pérnio ${ }^{1,9,12,19}$ e $4 \%$ dos pacientes (21/524) apresentaram outros

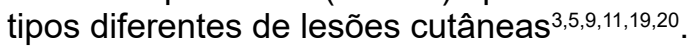

A localização das lesões cutâneas foi descrita em $97 \%$ dos casos $(510 / 524)^{1-18,20}$, com a maioria das lesões, $85,6 \%$ (437/510) sendo encontradas em tronco e nos membros $3,6-8,10,12,14-18$. Além disso, $14,3 \%$ (73/510) dos pacientes apresentaram manifestações cutâneas em membros inferiores e extremidades ${ }^{1,2,4,5,9,11,13,20}$.

Além das lesões cutâneas, 90,65\% (475/524) dos pacientes apresentaram outros sintomas ${ }^{1-10,13-15,17}$, sendo a febre o sintoma descrito mais comum, presente em $13,2 \%$ (63/475) dos pacientes $2-4,6-8,10,13-15$. Seguido pelas manifestações gastrointestinais, a qual foi verificada em 7,57\% (36/475) dos pacientes ${ }^{1,5,9,10}$. Além disso, $2,4 \%$ (13/475) do restante dos casos sintomáticos relataram outras manifestações, como tosse seca, dispneia, anosmia e cefaleia ${ }^{3-5,8,13,14}$. Já os $9,35 \%$ $(49 / 524)$ dos casos restantes não descreveram a manifestação de outros sintomas ${ }^{11,12,16,18-20}$. É importante ressaltar que 86,7\% (412/475) desses pacientes não tiveram o sintoma de febre descrito ${ }^{1,5,9,17}$.

Apenas 10,6\% (56/524) dos casos obtiveram biopsia das lesões $2-5,7,10-12,14,20$, sendo a maioria $82,1 \%$ (46/56) definida como consistente para infecção viral $\left.\right|^{8,16}$.
Ainda, 17,8\% (10/56) das histologias descreveram infiltrados moderados linfocíticos na derme superficial, focos de espongiose e paraqueratose em epiderme, derme com vasos dilatados e presença de neutrófilos, extravasamento de glóbulos vermelhos e infiltrado linfocítico perivascular ${ }^{1,2,10,12,14,15}$.

O tratamento foi relatado em apenas $3 \%$ dos casos (16/524), sendo observado o uso de corticoide sistêmico isolado ou associado a anti-histamínico em 43,7\% (7/16) dos pacientes ${ }^{4,5,7,12}, 25 \%$ (4/16) dos pacientes fizeram o uso dos fármacos lopinavir/ ritonavir associado à hidroxicloroquina ${ }^{2,3}$ e $12,5 \%$ (2/16) usaram a combinação de hidroxicloroquina e azitromicina ${ }^{11}$. Por fim, 18,7\% (3/16) relataram o uso de outros fármacos para o tratamento dos sintomas ${ }^{14,20}$. Assim sendo, 96,1\% (504/524) dos pacientes não tiveram a conduta do tratamento descrita $^{1,3,6,8,9,13,15,16-19}$.

\section{DISCUSSÃO}

Nesta revisão procuramos demonstrar, com base em dados obtidos de diversos estudos, a importância de se conhecer as manifestações cutâneas mais prevalentes que possam ser ocasionadas pelo COVID-19. Desse modo, facilitando aos médicos a abordagem de pacientes com a doença, com o intuito de melhorar o reconhecimento e diagnóstico de casos com poucos sintomas, ainda mais nesta fase inicial na qual há muitas incertezas. Uma característica importante da nossa revisão é que a maioria das descrições das lesões foram feitas por especialistas com base apenas na morfologia. Também, dado o número razoável de participantes e sua distribuição, é provável que a amostra seja representativa da distribuição geral das lesões cutâneas do COVID-1917.

Observamos cinco principais tipos de manifestações cutâneas nos estudos utilizados. Em ordem decrescente de prevalência são elas: exantema maculopapular generalizado morbiforme, erupção cutânea papulovesicular, urticária, livedo reticular e placas eritematosas. Esses padrões aparecem em momentos diferentes da doença e estão associados a diferentes durações, gravidade e provavelmente prognóstico. Vale destacar também, que grande parte das lesões foram encontradas em tronco e extremidades. Além disso, como nossa revisão incluiu lesões cutâneas inexplicáveis em pacientes com COVID-19, é possível que algumas delas tenham causas alternativas e, como essas lesões aparecem mais tarde na evolução e são menos comumente associadas à confirmação virológica, é possível que não estejam relacionadas à doença. Porém, alguns indícios falam a favor, como as lesões do tipo eritema pérnio que apresentaram elevada incidência em períodos de clima quente e 
os pacientes frequentemente tiveram contato com o COVID-19. Apenas um de 71 pacientes tinham histórico anterior de frieira. Também, seguindo neste aspecto, as lesões livedóides e necróticas podem ser lesões primárias do COVID-19 ou simplesmente indicar complicações que levam à oclusão vascular, uma vez que o COVID-19 tem sido associado a alterações na coagulação e dano vascular ${ }^{17}$.

Publicações anteriores descreveram alguns desses padrões de manifestações cutâneas aqui citados, mas são baseadas em poucos casos. Segundo a literatura anterior e a própria experiência na área médica em relação a manifestações cutâneas de doenças virais, é incomum que um único vírus possa levar a vários padrões clínicos diferentes, especialmente porque diferentes padrões não coexistem no mesmo paciente. Pacientes que talvez sejam classificados como tendo mais de um padrão são muito incomuns. Uma hipótese para explicar esse polimorfismo talvez seja que alguns deles tenham causas alternativas ou que haja diferenças no vírus ou no hospedeiro ${ }^{17}$.

O presente artigo apresentou limitações durante a revisão dos casos, como o número reduzido de biópsias das lesões analisadas e escassez de fotos que as caracterizem. Ademais, os estudos descritos utilizam diversas nomenclaturas para as lesões, fato que dificulta a classificação. Além disso, uma parcela dos pacientes não apresentou exame positivo para o vírus, somente suspeita. Outrossim, o tratamento dos casos não foi exposto na maioria dos relatos. Também é importante considerar que algumas lesões de pele podem ter causas alternativas, bem como podem ser provocadas por reações medicamentosas, visto que esses casos clínicos geralmente são graves e acabam sendo submetidos a diversos esquemas de medicamentos ${ }^{17}$.

\section{CONCLUSÃO}

As manifestações cutâneas relacionadas à COVID-19 ocorrem em uma pequena parcela dos pacientes e ainda não é possível afirmar que esses achados estejam relacionados exclusivamente com o quadro infeccioso da SARS-Cov-2. Para tanto, são necessárias mais pesquisas robustas sobre o assunto, com confirmações diagnósticas e análise histológica das lesões, a fim de excluir outras causas que possam desencadear esse mesmo quadro cutâneo. Contudo, compreender as diversas apresentações da COVID-19 é de extrema importância no entendimento epidemiológico da doença, assim como para auxiliar no diagnóstico precoce dessa infecção. Em função disso, tanto médicos como demais profissionais da saúde devem atentar para tais manifestações em seus pacientes, que em conjunto com os demais sinais e sintomas sistêmicos ajudam a compor as características clinicas da doença, corroborando para um diagnóstico clínico e diferencial precoce.

\section{REFERÊNCIAS}

1. Locatelli AG, Robustelli TE, Vezzoli P, Carugno A, Moggio E, Consonni L, et al. Histologic features of long-lasting chilblain-like lesions in a pediatric COVID-19 patient. J Eur Acad Dermatol Venereol. 2020;34(8):365-8.

2. Diaz-Guimaraens B, Dominguez-Santas M, Suarez-Valle A, Pindado-Ortega C, Selda-Enriquez G, Bea-Ardebol S, et al. Petechial Skin Rash Associated with Severe Acute Respiratory Syndrome Coronavirus 2 Infection. JAMA Dermatol. 2020;156(7):820-2.

3. Sachdeva M, Gianotti R, Shah M, Bradanini L, Tosi D, Veraldi S, et al. Cutaneous manifestations of COVID-19: Report of three cases and a review of literature. J Dermatol Sci. 2020;98(2):75-81.

4. Henry D, Ackerman M, Sancelme E, Finon A, Esteve E. Urticarial eruption in COVID-19 infection. J Eur Acad Dermatol Venereol. 2020;34(6):244-5.
5. Estébanez A, Pérez-Santiago L, Silva E, Guillen-Climent S, García-Vásquez A, Ramón MD. Cutaneous manifestations in COVID-19: a new contribution. $J$ Eur Acad Dermatol Venereol. 2020;34(6):250-1.

6. Hunt M, Koziatek C. A Case of COVID-19 Pneumonia in a Young Male with Full Body Rash as a Presenting Symptom. Clin Pract Cases Emerg Med. 2020;4(2):219-21.

7. Moreno RA, Villa LME, VA, Villa CE, Aparicio MAM, Fontanella JAA. Cutaneous manifestation of COVID-19 in images: a case report. $J$ Eur Acad Dermatol Venereol. 2020;34(7):307-9.

8. Marzano AV, Genovese G, Fabbrocini G Pigatto P, Monfrecola G, Piraccini BM, et al. Varicella-like exanthem as a specific COVID-19-associated skin manifestation: Multicenter case series of 22 patients. J Am Acad Dermatol. 2020;83(1):280-5.
9. Piccolo V, Neri I, Filippeschi C, Oranges T, Argenziano G, Battarra VC, et al. Chilblain-like lesions during COVID-19 epidemic: a preliminary study on 63 patients. J Eur Acad Dermatol Venereol. 2020;34(7):291-3.

10. Rivera-Oyola R, Koschitzky M, Printy R, Liu S, Stanger R, Golant $A K$, et al. Dermatologic findings in 2 patients with COVID-19. JAAD Case Rep. 2020;6(6):537-9.

11. Janah H, Zinebi A, Elbenaye J. Atypical erythema multiforme palmar plaques lesions due to Sars-Cov-2. $J$ Eur Acad Dermatol Venereol. 2020;34(8):373-5.

12. Jimenez-Cauhe J, Ortega-Quijano D, Carretero-Barrio I, Suarez-Valle A, Saceda-Corralo D, Moreno-Garcia del Real C, et al. Erythema multiforme-like eruption in patients with COVID-19 infection: clinical and histological findings. Clin Exp Dermatol. 2020;45(7):891-921. 
13. Manalo IF, Smith MK, Cheeley J, Jacobs R. A dermatologic manifestation of COVID-19: Transient livedo reticularis. J Am Acad Dermatol. 2020;83(2):700.

14. Ahouach B, Harent $S$, Ullmer A, Martres $P$, Bégon E, Blum L, et al. Cutaneous lesions in a patient with COVID-19: are they related? $\mathrm{Br} \mathrm{J}$ Dermatol. 2020;183(2):31.

15. Sanchez A, Sohier P, Benghanem S, L'honneur A, Rozenberg F, Dupin N, et al. Digitate Papulosquamous Eruption Associated with Severe Acute Respiratory Syndrome Coronavirus 2 Infection. JAMA Dermatol. 2020;156(7):819-20.
16. Fernandez-Nieto $D$, Ortega-Quijano $D$, Jimenez-Cauhe J, Burgos-Blasco $P$, Perosanz-Lobo D, Suarez-Valle A, et al. Clinical and histological characterization of vesicular COVID-19 rashes: A prospective study in a tertiary care hospital. Clin Exp Dermatol. 2020;45(7):872-5.

17. Galvan Casas $C$, Català $A$, Carretero Hernández $G$, Rodríguez-Jiménez $P$, Fernández-Nieto A, RodríguezVilla Lario A, et al. Classification of the cutaneous manifestations of COVID-19: a rapid prospective Nationwide consensus study in Spain with 375 cases. Br J Dermatol. 2020;183(1):71-7.
18. Tammaro A, Adebanjo GAR, Parisella FR, Pezzuto A, Rello J. Cutaneous manifestations in COVID-19: the experiences of Barcelona and Rome. $J$ Eur Acad Dermatol Venereol. 2020;34(7):306-7.

19. Bouaziz JD, Duong T, Jachiet M, Velter C, Lestang P, Cassius C, et al. Vascular skin symptoms in COVID-19: a French observational study. JEADV. 2020;34(9):451-2.

20. Castelnovo L, Capelli F, Tamburello A, Faggioli PM, Mazzone A. Symmetric cutaneous vasculitis in COVID-19 pneumonia. J Eur Acad Dermatol Venereol. 2020;34(8):362-3.

Recebido: 17 ago, 2020 Aceito: $14 \mathrm{dez}, 2020$ 